\title{
Leucoplasia Verrucosa Proliferativa: A Propósito de un Caso Clínico
}

\author{
Proliferative Verrucous Leukoplakia: A Clinical Case
}

\author{
Alejandra Fernández Moraga*; Maureen Marshall* \& Alfredo Esguep Sarah ${ }^{* *}$
}

FERNÁNDEZ, M. A.; MARSHALL, M. \& ESGUEP, S. A. Leucoplasia verrucosa proliferativa: a propósito de un caso clínico. Int. J. Odontostomat., 7(3):379-383, 2013.

RESUMEN: La Leucoplasia Verrucosa Proliferativa (LVP) es una placa blanca, verrucosa, exofítica, de crecimiento lento, que no se desprende al raspado y que tiende a comprometer diversos sitios de la mucosa oral. Es resistente al tratamiento independiente de la terapia adoptada y presenta una alta tasa de recidiva posterior al tratamiento quirúrgico. Desde 1985, cuando fue descrita por primera vez por Hansen, hasta le fecha, se ha mantenido como una entidad patológica compleja. Reportamos un caso de una paciente de sexo femenino de 72 años de edad que consulta por una lesión blanca, verrucosa, asintomática, que no se desprende al raspado, en el borde y vientre lingual y piso de boca. Se realizaron 2 biopsias incisionales del borde lateral de la lengua. Al examen microscópico se observó en ambas biopsias un grueso revestimiento epitelial acantósico, con marcada hiperparaqueratosis, una membrana basal nítida y cambios displásicos mínimos. Al mes de realizadas las biopsias la lesión presentó recidiva, manteniendo las mismas características clínicas encontradas inicialmente. Considerando la evolución, comportamiento biológico y las características histopatológicas, la lesión se diagnosticó como una LVP. Hasta la fecha, no hay protocolo de tratamiento establecido para estas lesiones. En este caso en particular, tomando en cuenta la extensión de la lesión, los sitios comprometidos, las escasas atipias presentes al examen histopatológico, la edad y escaso nivel socioeconómico de la paciente, se decidió no realizar la remoción quirúrgica de la lesión y mantenerla en control clínico cada 3 meses. Después de varios controles realizados durante un periodo de 3 años, se observó que la lesión mantuvo sus características clínicas iniciales. Esto confirma que haber optado por un tratamiento conservador, en este caso en particular de LVP, fue la decisión correcta. Sin embargo, es importante considerar que, según lo reportado en la literatura, la tasa de transformación maligna es alta e impredecible, por lo que los controles clínicos periódicos son de vital importancia para diagnosticar precozmente su evolución a carcinoma espino celular o carcinoma verrucoso.

PALABRAS CLAVE: Leucoplasia Verrucosa Proliferativa, leucoplasia oral, patología oral.

\section{INTRODUCCIÓN}

La leucoplasia verrucosa proliferativa (LVP) fue descrita por primera vez por Hansen et al. (1985) como un tipo específico de leucoplasia oral que presenta características clínicas distintivas: un comportamiento biológico agresivo con tendencia a la multifocalidad, alta tasa de recurrencia después del tratamiento, y de transformación maligna, pudiendo progresar ya sea a un Carcinoma Verrucoso (CV) o a un Carcinoma Espino Celular (CEC) (Bagan et al., 2010).

Inicialmente, la LVP se observa como una placa blanca que no se desprende al raspado, de crecimiento lento, que con el tiempo se vuelve verrucosa y exofítica, pudiendo tener áreas eritematosas y llegando a comprometer diversas partes de la cavidad bucal, como el paladar duro y blando, mucosa alveolar, lengua, piso de boca, encía y el labio (Hansen et al.; Cabay et al., 2007; Cerero-Lapiedra et al., 2011).

Las mujeres se ven más afectadas que los hombres, sobre todo después de los 60 años (Cabay et al.). No se ha visto que exista una predilección racial (Ghazali et al., 2003) y, a pesar de los diversos intentos por asociarla a un factor determinado como el tabaco o el Virus Papiloma Humano (VPH), aún no se ha podido detectar una relación válida con ninguno de ellos.

* Cirujano Dentista, Docente Cátedra de Patología, Universidad Andrés Bello, Viña del Mar, Chile.

**Profesor Titular Departamento de Cirugía y Patología, Facultad de Odontología, Universidad Andrés Bello, Viña del Mar, Chile. 
Desde 1985 hasta la fecha, la LVP se ha mantenido como una entidad patológica compleja. Su etiología aún sigue siendo desconocida (van der Waal \& Reichart, 2008). Es de difícil diagnóstico, tanto clínico como histopatológico, incluso para ojos expertos, ya puede confundirse con una leucoplasia homogénea, leucoplasia verrucosa, eritroleucoplasia, carcinoma verrucoso, liquen plano, entre otras (Hansen et al.). No existe un consenso sobre criterios diagnóstico o protocolos de tratamiento aceptados, lo que hace difícil establecer comparaciones entre los diversos estudios publicados en la literatura (van der Waal \& Reichart; Bishen \& Sethi, 2009). Con el fin de aportar al diagnóstico diferencial de las lesiones verrucosas, su manejo y evolución, en el presente estudio reportaremos y discutiremos un caso de LVP.

\section{REPORTE DE CASO}

Paciente de sexo femenino de 72 años de edad fue derivada desde el Servicio de Odontología del Consultorio Alcalde Manríquez, Quilpué,Chile, al servicio de Patología y Medicina Oral de la Facultad de Odontología de la Universidad Nacional Andrés BeIlo, Viña del Mar, Chile. La paciente no tenía antecedentes mórbidos relevantes, ni hábitos asociados como tabaco y alcohol.

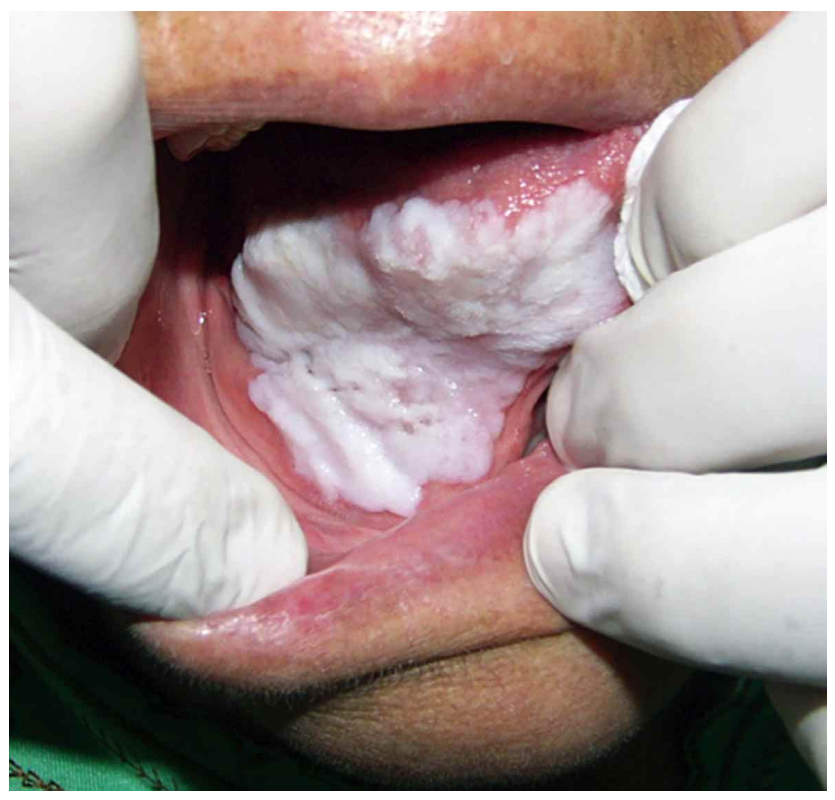

Fig. 1. Extensa lesión blanca, de superficie verrucosa, que no se desprende al raspado que compromete el borde y vientre de la lengua y piso de boca.
Al examen intraoral se observó una extensa lesión blanca, que no se desprendía al raspado, de superficie verrucosa, que se encontraba comprometiendo el borde y vientre lingual y piso de boca (Fig. 1). Se realizó una biopsia incisional del borde y vientre lingual.

Al examen microscópico con hematoxilina eosina se observó un grueso revestimiento epitelial acantósico, con marcada hiperparaqueratosis y una membrana basal nítida. Las células epiteliales presentaban algunos cambios como hipercromatismo nuclear, hiperplasia del estrato de células basales, degeneración vacuolar y algunas papilas en forma de gota. No se observó tejido conectivo subepitelial.

En el control al mes fue posible observar focos de recidiva en el área de la biopsia (Fig. 2). Al mes se observó la recurrencia total de la lesión. Se decidió hacer una prueba de Reacción de Polimerasa en Cadena (PCR) para detección de Virus Papiloma Humano (VPH), el cuál resultó negativo, y realizar una segunda biopsia incisional de la misma zona. En el examen histopatológico se observó lo mismo que en la primera muestra, con un moderado infiltrado inflamatorio crónico en el tejido conjuntivo subepitelial (Fig. 3).

Al mes de realizada la segunda biopsia, se observa una nueva recidiva en la misma zona. Considerando la evolución clínica, examen histopatológico y basándonos tanto en la definición de Hansen et al. como en los criterios de Cerero-Lapiedra et al. (2011), la lesión se consideró una LPV.

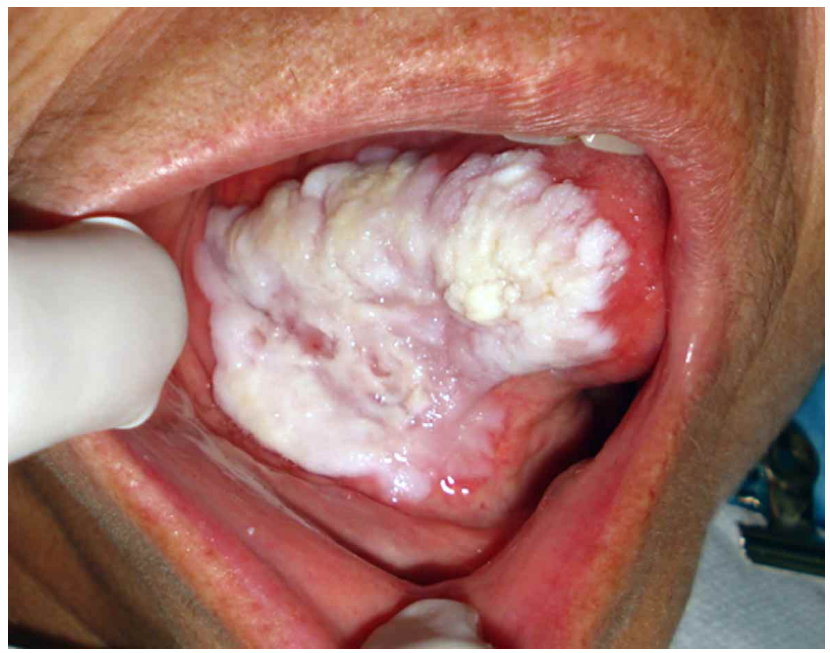

Fig. 2. Un mes después de realizada la biopsia incisional fue posible observar la recurrencia total de la lesión. 


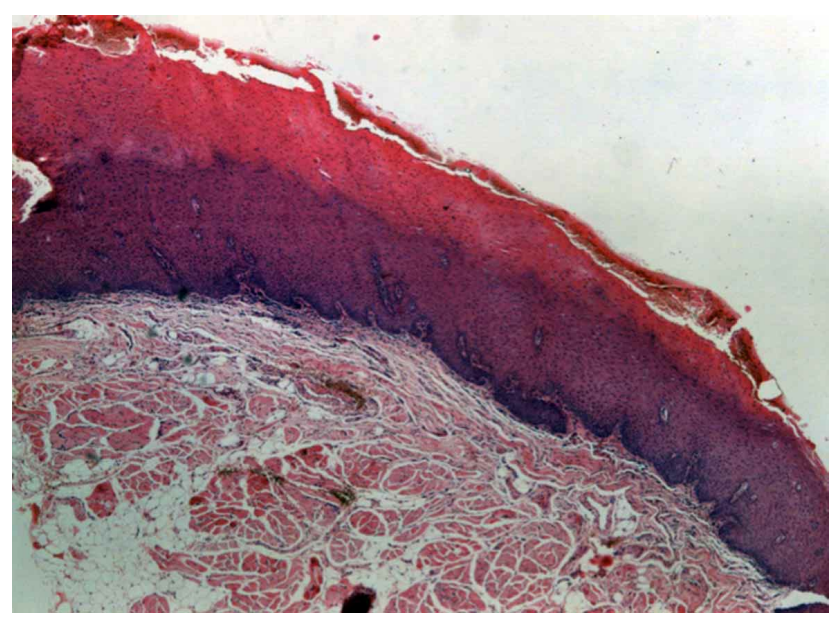

Fig. 3. Al examen microscópico con tinción de hematoxilina eosina se observa un grueso y acantósico revestimiento epitelial con una marcada hiperparaqueratosis y mantención de la membrana basal (40X).

\section{DISCUSIÓN}

Durante mucho tiempo el diagnóstico de LVP se ha realizado tanto desde un punto de vista clínico como histológico basado en la primera definición hecha por Hansen en 1985. Pero nosotros nos cuestionamos, al igual que otros autores como Gahazali et al., Van del Waal \& Reichart y Bishen \& Sethi, si realmente todos los casos publicados en la literatura como LVP realmente lo eran. Es por esta razón que en el 2010 Cerero-Lapiedra et al. propusieron una serie de criterios diagnósticos específicos para la LVP (Tabla I). Considerando lo establecido tanto por Hansen et al. como por Cerero-Lapiedra et al., este caso cumple con todos los requisitos para ser considerada una LVP.
Clínicamente corresponde a una leucoplasia mayor a $3 \mathrm{~cm}$, de superficie exofítica y verrucosa, que se encontraba comprometiendo más de un sitio de la cavidad oral: el borde y vientre lingual y piso de boca. La lesión recidivó no sólo una, sino que dos veces después de realizadas las biopsias incisionales. Histopatológicamente, la lesión era compatible con una LVP grado 4 según Hansen (esto correponde a hiperqueratosis con o sin displasia epitelial leve y clínicamente hiperplasia verrucosa), con cambios displásicos mínimos como hipercromatismo nuclear, hiperplasia del estrato basal y papilas en forma de gota, que no justifican un comportamiento tan agresivo.

Con respecto a posibles agentes etiológicos, hasta la fecha no ha sido posible encontrar ninguno que se asocie con la LVP. Diversos estudios realizado intentado establecer la asociación entre LVP con Candida albicans (Silverman \& Gorsky, 1997), virus Epstein Barr (Horiuchi et al., 1995) y VPH (Silverman \& Gorsky; Eversole, 2000), específicamente con los de potencial oncogénico como los tipos 16 y 18, expresión de factor de crecimiento transformante alfa (Kannan et al., 1996), p53, Ki-67, Mcm-2 y Mcm-5 (Gouvea et al., 2010). Todos presentaron resultados poco concluyentes. En el presente caso se realizaron pruebas para detección de Candida albicans y presencia de VPH, las cuales fueron negativas. No se hicieron estudios para otros posibles agentes etiológicos.

El paciente afectado era de género femenino, sobre 70 años de edad, sin factores de riesgo asociados como el tabaco y alcohol. Todas estas son características que coinciden con casos similares de LVP reportados en la literatura a los largo de los años (Cabay et al.; Gazhali et al.; van der Waal \& Reichart).

Tabla I. Criterios mayores y menores para diagnóstico de LVP (Cerero et al., 2011).

\begin{tabular}{|c|c|}
\hline Criterios mayores (MC) & Criterios menores (mc) \\
\hline $\begin{array}{l}\text { A. Una leucoplasia con compromiso de más de } 2 \\
\text { sitios diferentes d la cavidad oral, los cuales } \\
\text { generalmente son la encía, proceso alveolar y } \\
\text { paladar. }\end{array}$ & $\begin{array}{l}\text { Leucoplasia oral que mida más de } 3 \mathrm{~cm} \text {, sumando } \\
\text { todas las áreas afectadas. }\end{array}$ \\
\hline B. Existencia de un área verrucosa. & El paciente debe ser de sexo femenino. \\
\hline $\begin{array}{l}\text { C. Las lesiones deben haberse engrosado } \\
\text { diseminado durante la evolución de la lesión. } \\
\text { D. Debe existir una recurrecia previa de la lesión. }\end{array}$ & $\begin{array}{l}\text { El paciente (mujer u hombre) debe ser no-fumador. } \\
\text { La evolución de la lesión debe ser mayor a } 5 \text { años. }\end{array}$ \\
\hline $\begin{array}{l}\text { E. Histopatológicamente, puede corresponder a una } \\
\text { hiperqueratosis simple, hiperplasia verrucosa, } \\
\text { carcinoma verrucoso o carcinoma espino celular, ya } \\
\text { sea insitu o infiltrativo. }\end{array}$ & \\
\hline
\end{tabular}


Hasta la fecha, aún no existe un protocolo de tratamiento establecido para la LVP. La literatura nos muestra que para combatir este tipo de lesión el uso de radioterapia, quimioterapia y cirugía láser no son plenamente satisfactorias (Gouvea et al.; Lodi \& Porter, 2008). Por esto se aconseja realizar un tratamiento quirúrgico, a pesar de caracterizarse por recidivar posterior a éste. Sin embargo en este caso, al considerar la extensión de la lesión que presentaba la paciente, que era de $3 \mathrm{~cm}$, los sitios que afectaba, borde y vientre lingual y piso de boca, las escasas atipías encontradas en el examen histopatológico que señalaban que no había indicios de malignidad, la avanzada edad y su bajo nivel socioeconómico, se determinó, en conjunto con sus familiares, mantenerla bajo control clínico cada 3 meses. Tanto la paciente como su familia fueron instruidos en el sentido que ante cualquier cambio de la lesión como ulceración, erosión, crecimiento y/o sangramiento, deberían acercarse al servicio de Patología y Medicina Oral de la Facultad de Odontología de la Universidad Nacional Andrés Bello, Viña del Mar. Después de varios controles realizados en un período de 3 años, pudimos observar que la lesión presenta las mismas características clínicas que al examen inicial. Esto corrobora que nuestra decisión de tener una manejo conservador para éste caso en particular de LPV fue acertado. No obstante, hay que considerar que según lo reportado en la literatura, el riesgo que la LVP se malignice es impredecible, por lo que el control clínico periódico es de suma importancia para pesquisar cualquier cambio sugerente de transformación a CEC o CV.

En vista de todo lo anterior mencionado, creemos que es importante tener en cuenta los cambios clínicos en la mucosa oral sugerentes de LVP, a pesar de su baja frecuencia. Una vez establecido su diagnóstico, posterior a exámenes tanto clínico como histopatológico, aconsejamos evaluar las características propias de cada lesión y paciente antes de establecer un plan de tratamiento definitivo, ya que en nuestro caso no fue necesario uno quirúrgico y pudo ser manejado exitosamente de manera conservadora con un estricto control clínico periódico a fin de identificar, en caso que suceda, cambios clínicos sospechosos de malignización. Es aconsejable educar al paciente en la importancia de asistir a los correspondientes controles, además de enseñarle el autoexamen oral para detectar tempranamente posibles cambios en la textura y color de la mucosa.

FERNÁNDEZ, M. A.; MARSHALL, M. \& ESGUEP, S. A. Proliferative Verrucous Leukoplakia: a clinical case. IInt. J. Odontostomat., 7(3):379-383, 2013.

ABSTRACT: The proliferative verrucous leukoplakia (PVL) is a white plate, wart-like, exophytic, slow growth , which does not follow the scaling that tends to compromise various oral mucosal sites . It is resistent to independent therapy and has a high rate of recurrence after surgical treatment. Since 1985, when it was first described by Hansen, and to date it has remained a complex pathological disorder. We report a case of a 72-year-old female patient who complains of a white, verrucous and asymptomatic lesion. The lesion does not detach by scraping the edge, lingual belly or mouth floor. Two Incisional biopsies were performed on lateral border of the tongue. Upon microscopic examination of both biopsies, thick epithelial lining, with marked hyperparakeratosis, a basement membrane, and minimal dysplasia alterations were observed. One month following biopsies of the lesion were performed, lesion relapsed and maintained the same clinical features found initially. Considering the evolution, biological behavior and histopathological features, the lesion is diagnosed as an PVL. To date, there is no established treatment protocol for these injuries. In this particular case, taking into account the extent of the injury, compromised sites, the few atypia present histopathological examination, age and low socioeconomic level of the patient, it was decided not to perform surgical removal of the lesion and maintain clinical monitoring every 3 months. Following several examinations carried out over a three-year period, it was observed that the injury kept initial clinical features. This confirms that having opted for conservative treatment, in this particular case of PVL, was the right decision. However, it is important to consider that, as reported in the literature, there is a high and unpredictable rate of malignant transformation. Therefore periodic clinical controls are vital for early diagnosis of cell carcinoma or verrucous carcinoma.

KEY WORDS: proliferative verrucous leukoplakia, oral leukoplakia, oral pathology.

\section{REFERENCIAS BIBLIOGRÁFICAS}

Bagan, J.; Scully, C.; Jimenez, Y. \& Martorell, M. Proliferative verrucous leukoplakia: A concise update. Oral Dis., 16(4):328-32, 2010.
Bishen, K. A. \& Sethi, A. Proliferative verrucous leukoplakia-diagnostic pitfalls and suggestions. Med. Oral Patol. Oral Cir. Bucal, 14(6):E263-4, 2009. 
FERNÁNDEZ, M. A.; MARSHALL, M. \& ESGUEP, S. A. Leucoplasia verrucosa proliferativa: a propósito de un caso clínico. Int. J. Odontostomat., 7(3):379-383, 2013.

Cabay, R. J.; Morton, T. H. Jr. \& Epstein, J. B. Proliferative verrucous leukoplakia and its progression to oral carcinoma: a review of the literature. J. Oral Pathol. Med., 36(5):255-61, 2007.

Cerero-Lapiedra, R.; Baladé-Martínez, D.; Moreno-López, L. A.; Esparza-Gómez, G. \& Bagán, J. V. Proliferative verrucous leukoplakia: a proposal for diagnostic criteria. Med. Oral Patol. Oral Cir. Bucal, 15(6):e839-45, 2011.

Eversole, L. R. Papillary lesions of the oral cavity: relationship to human papilloma viruses. J. Calif. Dent. Assoc., 28(12):922-7, 2000.

Ghazali, N.; Bakri, M. M. \& Zain, R. B. Aggressive, multifocal oral verrucous leukoplakia: Proliferative verrucous leukoplakia or not? J. Oral Pathol. Med., 32(7):383-92, 2003.

Gouvea, A. F.; Vargas, P. A.; Coletta, R. D.; Jorge, J. \& Lopes, M. A. Clinicopathological features and immunohistochemical expression of p53, Ki-67, Mcm-2 and Mcm-5 in proliferative verrucous leukoplakia. J. Oral Pathol. Med., 39(6):447-52, 2010.

Hansen, L. S.; Olson, J. A. \& Silverman, S. Jr. Proliferative verrucous leukoplakia. A long-term study of thirty patients. Oral Surg. Oral Med. Oral Pathol., 60(3):285-98, 1985.

Horiuchi, K.; Mishima, K.; Ichijima, K.; Sugimura, M.; Ishida, T. \& Kirita, T. Epstein-Barr virus in the proliferative diseases of squamous epithelium in the oral cavity. Oral Surg. Oral Med. Oral Pathol. Oral Radiol. Endod., 79(1):57-63, 1995.

Kannan, R.; Bijur, G. N.; Mallery, S. R.; Beck, F. M.; Sabourin, C. L.; Jewell, S. D.; Schuller, D. E. \& Stoner, G. Transforming growth factor-alpha overexpression in proliferative verrucous leukoplakia and oral squamous cell carcinoma: an inmunohistochemical study. Oral Surg. Oral Med. Oral Pathol. Oral Radiol. Endod., 82(1):6974, 1996.

Lodi, G. \& Porter, S. Management of potentially malignant disorders: evidence and critique. J. Oral Pathol. Med., 37(2):63-9, 2008.

Silverman, S. Jr. \& Gorsky, M. Proliferative verrucous leukoplakia: a follow-up study of 54 cases. Oral Surg. Oral Med. Oral Pathol. Oral Radiol. Endod., 84(2):1547, 1997.

van del Waal, I. \& Reichart, P. A. Oral proliferative leukoplakia revisited. Oral Oncol., 44(8):719-21, 2008.

\author{
Dirección para Correspondencia \\ Alfredo Esguep Sarah \\ Avenida Valparaíso 1560 \\ Viña del Mar \\ CHILE
}

Teléfono: +569979986924

Email: aesguep@unab.cl

Recibido : 11-08-2013

Aceptado: 11-10-2013 